\title{
CONGESTION CONTROL FOR A ULTRA-WIDEBAND DYNAMIC SENSOR NETWORK USING AUTONOMIC BASED LEARNING
}

\author{
Sanjay K N \\ Research Scholar, Department of Electronics and Communication Engineering, \\ Visvesvaraya Technological University-Research Center, \\ Vivekananda Institute of Technology, India \\ Shaila K \\ Professor, Department of Electronics and Communication Engineering, \\ Vivekananda Institute of Technology, Bengaluru, India \\ Venugopal K R \\ Vice-Chancellor, Bangalore University, Bengaluru, India
}

\begin{abstract}
The physical conditions of the area of interest is being collected at the central location using a set of dedicated sensors that forms a network is referred to as Wireless Sensor Network. A dynamic environment is required for a secure multi-hop communication between nodes of the heterogeneous Wireless Sensor Network. One such solution is to employ autonomic based learning in a MAC Layer of the UWB TxRx. Over a time period the autonomic based network learns from the previous experience and adapts to the environment significantly. Exploring the Autonomicity would help us in evading the congestion of about $30 \%$ in a typical UWB-WSNs. Simulation results showed an improvement of 5\% using Local Automate Collision Avoidance Scheme (LACAS-UWB) compared to LACAS.
\end{abstract}

Key words: Autonomic Network Architecture, Dynamic En- vironment, LACAS, Stocastic Model, Ultra Wide-band, Wireless Sensor Networks.

Cite this Article: Sanjay K N, Shaila K, Venugopal K R, Congestion Control for a Ultra-Wideband Dynamic Sensor Network Using Autonomic Based Learning, International Journal of Computer Engineering and Technology 10(5), 2019, pp. 20-37.

http://iaeme.com/Home/issue/IJCET?Volume $=10 \&$ Issue $=5$ 


\section{INTRODUCTION}

In collaborative space-timing task requires a low cost integrated sensing, communicating and computing nodes that involves an innovative technology in wireless networking, array processing and microelectronics. In the areas of embedded systems, networking, multi-agent systems and pervasive computing WSN finds a significant consideration due to the real time scenarios like environment monitoring, disaster relief. With the combination of large number of static sensor nodes the distributive sensing would be achieved in WSNs [1].

The unique functioning of WSNs can be characterized and the effective use of the communication protocol itself is mandatory and demands for the cross-layer design. One such approach is to combine the Local Automate and Autonomic Network Architecture at the MAC level of the network [2]. This leads to self-healing network that are energy efficient MAC with self-organizing and fault-tolerant routing protocols that involves distributed algorithms.

MAC rules have been developed to minimize interference and packet collisions that includes [4], [5], [6]: optimizing the channel access, packet transmission and retransmission methods; packets lengths; modulation and coding; transmission powers; etc. are few of the well-known algorithms that are used till date [8], [9]. These techniques, are not well-suited to the WSNs due to the addressing issues. Thus, a serious paradigm shift in MAC designs is required.

Therefore, the decentralized character of a typical WSNs has to be rolled out that complicates with any number of attempts that are required to attain a network wide synchronization [10], [11]. Then, scheduling a data load in WSNs generally becomes very low: with the generated traffic in a highly directed data-processing sink unit's that exhibits a converge communication pattern. This needs special observation in the design process since, the nodes are close to the sink. The sink node has to manage more traffic with the available nodes in the perimeters using local automata [12], [13]. In WSNs MAC design two parameters play a vital role namely, number of nodes and number of two hop neighbor nodes. The processing capabilities is reduced due to low complexity of the nodes. While the average scheduling and end-to-end data reporting times are delayed because of limited buffer size [14].

The energy estimation is the largest design limitation of WSN for long network runtimes. In [15], the nodes are switched-off all the time irrespective of the intrinsic current leakage in the battery that limits the life-time upto 10-15 years depending on the operating temperature. This provides maximum network life-time with battery powered nodes.

In specific sensor applications, the utilization of energy is controlled by the node's radio consumption and in this particular case the network has to prefer UWB. It can be shown that when the node is in sleeping mode power consumption is negligible that enhances the life time since the radio is controlled by the MAC. Non-rechargeable battery; rechargeable battery with regular recharging (e.g. sunlight); rechargeable battery with irregular recharging (e.g. opportunistic energy scavenging); capacitive/inductive energy provision (e.g. active RFID); etc. [15] are few of the power mechanisms that are considered while increasing the life time of the battery. This has an influence on the choice and design of MAC protocol with local automata [16-18].

A large number of ultra-small autonomous UWB devices are considered in our work wherein the sensor node is equipped with the integrated sensors, data processing capabilities and short-range radio communications. The data communication between the nodes are 
forwarded to the specialized gateway nodes. Two alternative routing approaches have been considered for sensor networks namely, flat multi-hop and clustering [20-22].

The data communication between nodes are achieved via specialized gateway nodes using flat, multi-hop and clustering routing approaches. To minimize the costs the sensor data can be combined and compressed inside the node or cluster of nearby nodes and reduces the payload of the data packets [23]. The challenge is to manage the packet's overhead condition that is significant in WSN. The existing approaches mainly focusses on routing and destination identification issues [25]. A critical problem still exists that is the overhead of the MAC header or the MAC address. In current approach the unique identifiers are used which are of same size or larger than packet payload that shows the important source of energy consumption. The free space available in the cellular system contains the address agnostic and the addresses that are present in the data packets. This approach for the MAC addressing in the sensor network results in the energy savings [26-30].

The ANA architecture consisting of two layers of co-ordination namely, lower task execution layer and higher task allocation layer as shown in Figure 1. The dotted line shows the detail Autonomic Network Architecture that differs from existing layered architectures with multi-hop coordination that adopts a reactive method [31]. The following characteristics are exhibited while employing ANA with LACAS systems:

- Self-configuring: The sensor netwoks are made adaptive for the dynamic changing

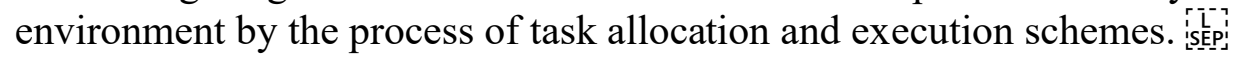

- Self-optimizing: In terms of topology, propagation and interference, the system configurations are autonomously and continually adapted to the traffic profile and network environment.

- Self-healing: The system of task distribution is robust to the network failures where task execution is capable of self-repairing unexpected robost formation damage.

- Self-protecting: The task performance allows the system to route and negotiate the complicated unforeseen barriers.

- Task Allocation for nodes: In the extreme case, the movement of nodes will be restricted due to the less work or no work involved. Thus, the general system performance is adversely affected by a task interference. To minimize the physical interference, in our work the nodes are distributed dynamically.

- Complexity of dynamically changing nodes: Existing nodes tend to underuse the sensor inputs that may provide helpful data to coordinate behaviors and choose the most suitable action.

- Coalition Formation for Minimalist nodes: Exciting multiagent formation schemes requires complex planning, particular formation schemes that require complex scheduling, explicit negotiation and precise coalition cost evaluation. Thus, they may not be able to operate in actual time in a large-scale sensor network.

- Cooperation of Resource-Constrained nodes: Only local, uncertain environmental information with limited communication and sensing capabilities can be obtained from sensor nodes. 


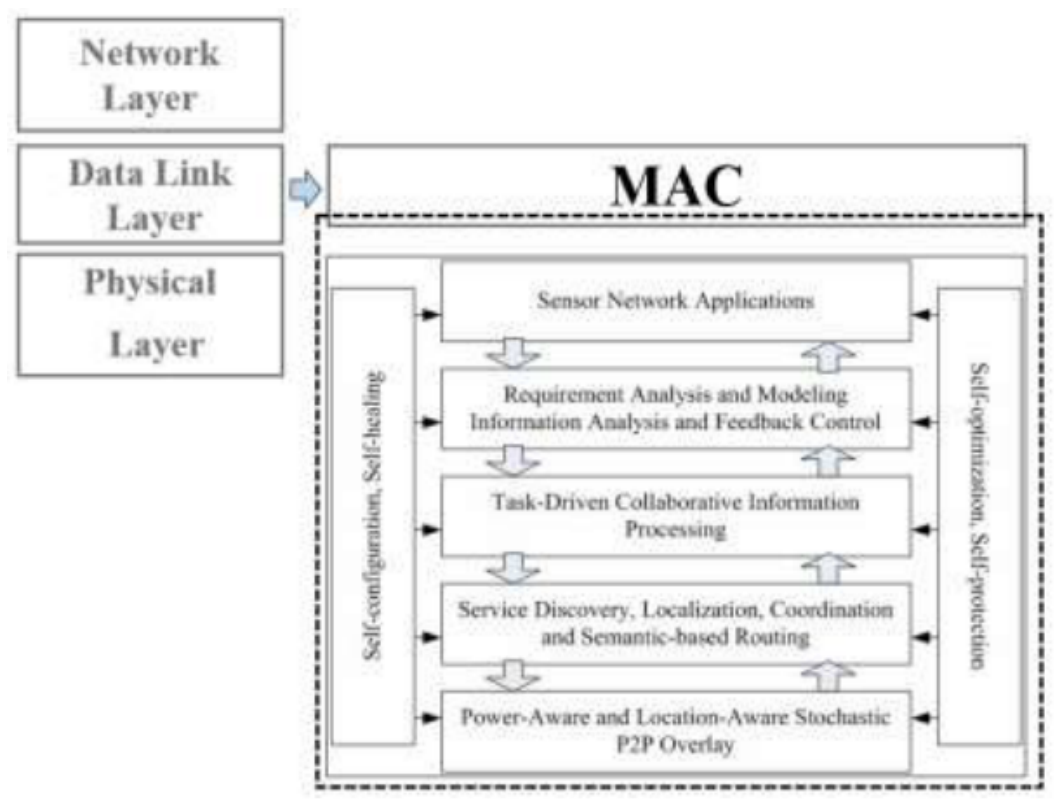

Figure 1. ANA: Autonomic Network Architecture

\section{Motivation}

The focus of the WSNs design is to provide the assurance of its long existence under specific energy and complexity constraints. The MAC plays an important role in this design because it has control on the active and sleeping state of each node. Therefore, MAC protocols needs the primary design factors like reliability, longevity, fairness, scalability and latency [6].

Congestion is the most important factor to be considered to achieve the good reliability of transmission of data in the network. Due to the congestion in WSN's traffic increases, that leads to dissipation of energy in colossal amount in the sensor nodes. This results in loss of packets and it creates unfair and non-reliable flow of packets [11]. In many cases nodes are made to work without any interruption for long durations without replacing the energy sources. Therefore, the key concern of WSNs is to optimize the energy consumption in sensor node [15]. With the help of intermediate nodes and localized control centres all the nodes in a sensor network transmit the data to the sink node [18]. This increases the possibility of congestion at the intermediate nodes. These issues have to be addressed while designing WSNs for any applications [19-20].

\section{Contribution}

To achieve reliable communication between the nodes at larger rate a Learning based Autonomic Network Architecture is proposed that integrates the UWB sensor network with the complex dynamic environments. The throughput and fault tolerance levels have to be optimized in dynamic environment containing large number of nodes. Thus, learning based autonomic network architecture in the MAC layer would provide a solution in managing this large sensor activity.

The introduction of LA based ANA could provide a continued communication even if the nodes are not in the communication range availing the function of Master/Slave to the respective nodes. 


\section{Organisation}

Section II provides a literature survey. Section III provides the basics of the work. The problem definition along with statement of the problem and objectives are discussed in Section IV with few assumptions. The system and mathematical model describes the mathematical model used for the implementation of LA-ANA based architecture in Section V. Section VI provides the simulation environment and the performance metrics used in evaluating the results.

\section{LITERATURE SURVEY}

The evolution in the WSNs which adapts dynamically to the environment is developed in [1] that uses the reconfigurable WSN/RWiN Meta model based CrA and ZA architecture. This is a multi-agent architecture with RWSN meta-model and package with UML. The Routing table parameters and verification of temporal constraints are used to optimize the distribution of configuration with energy levels that controls the problem complexity and their temporal properties. The disadvantage of using the automatic adaptation of zones lead to the modification in their sizes for the larger and medium networks.

The dead lock and cross-layer verification process for the cache coherence protocol and the communication fabric is described in [2]. The automated study to obtain cross layer invariance are related to the application layer protocol. The XMAS and IO Automata, Artificial Protocol, Cross-layer deadlock and verification times are used to fine-grain the modelling of communication fabrics. These are the combined XMAS primitives with IO state automata which are applicable to communication fabrics that include routing, virtual channels, arbitration, broadcasting, adaptive routing etc. Here, the IO automata models are only suitable for the Gem5 description.

The complexity management and elevated security issues are found in Autonomic Intelligent Cyber-Sensor. To carry forward the Industrial Control Network there should be presence of Autonomic computing that enables the autonomic design along with the anomaly detection and design. Todd et. al., [3] used the Autonomic architecture and Simple object access protocol for designing the flexible two-level communication layer that can be used for anomaly detection and performance with sensor CPU utilization. The three complimentary module can be found in the dynamically service oriented architectures due to the ever changing environment. While the autonomous systems are exemplified with the Interference aware anticipatory algorithm and dynamic host protocol components which lags the integration of the intelligent energy efficient clustering (AICS) with external components utilizing the IF-MAP standard.

The Large-scale industrial system that relies on the operation for distributed controller uses the real distributed protocols that are basically designed using the shared-medium industrial communication protocols as described in [4]. This model uses several models like TIOA model and TSIL layer with URT protocol that are used to calculate analytical delay experienced by the RT messages and is limited to Ethernet.

During static optimizations with the assumed network characteristics has to be taken care to avoid the poor performance of the network. Hence, a joint routing and a power control for delay-sensitive is required in a multi-hop network. Cohen et. al., [5] provided a solution for the above said problem by autonomic and distributed joint routing process that involves Markov decision process. This maximizes the network utility in a dynamic environment with time-varying channels. The formulation, optimization and complexity of the problem is centralized and distributed with an actor-critic learning, that helps in the distributed computation method for finding optimal policy. The average throughput with and without overhead can be easily determined while the information exchange overhead is calculated. 
Peter Bohm et. al., [6] introduced a technology that involves a high-performance protocol implemented with the sophisticated features that meets the technological demands with an verified modelling of the PCI Express model. The PCI express transaction and data link layer are used in finding the generic interface and state machine wrapper for polymorphic buffers. The multiplex and arbitrate transformation with a simple model provides the sophisticated features that can be added incrementally using the generic transformation. While it is difficult to the user for showing a technical feasibility of a step-wise model deviation process.

Characterized by the energy-constraint nodes that are tasked with collecting and forwarding environmental parameters with spatial and temporal fidelity that requires an expedient wireless sensor automation as discussed in [7]. The system must be designed with the scalability and efficiency benefits. This model comprises automation architecture with a control strategy that includes ACK feedback automation, analytical results, variance of the number of sensors and recommending operating region, average turn time, standard deviation, scaling of $\mathrm{E} / \mathrm{Q}$. The architecture is expedient, scalable and does not require initialization of nodes while the cluster head nor the sensor nodes require explicit knowledge of $\mathrm{N}$.

The autonomic mobile sensor network discussed in [9] utilizes the self-coordinated allocation and execution of the regional distribution of tasks in proportion to that of the moving targets in a non-station environment. The behavioural coordination mechanism with conceptual description of EKMs helps in finding the adverse effects of task interference between the robots that can be minimized. The decomposition with the multi-agent systems in an autonomic task becomes difficult.

Thathachar et. al., [12] proposed the Learning algorithms for feed-forward connectionist system in reinforcement learning environment that enables the teams and hierarchies of the learning automata. The learning algorithm used is the LR-1 and the asymptotic of ordinary Differential Equations at the two levels of sub-units. The sub-unit of three automata, RMS error and probability of misclassification provides the local convergence properties and can handle context vectors with the global convergence.

In a spectrum access network the problem of distributed channel selection arises while minimizing the interference leading to a stochastic MAC-layer interference model [13]. This comprises of two models namely, stochastic MAC-layer interference model and Stochastic Learning-Automata based uncoupled algorithm SLA-H. The normalized throughput versus the distance between the links, CRN, convergence speed can be the exact potential game with pure strategy NE point at least defined WSN environment. While the randomness of channel fading gain in the transitional region is quite difficult.

The autonomous unmanned vehicle based networks with adversely situations in a minor fault in the system software/hardware is the Learning automata-based fault tolerant system. The AUxVs and cross-layer design with the learning automata is used in designing the dynamic autonomous unmanned vehicular networks as proposed in [14]. The UARC with the LCF model enhances the throughput with respect to pause time with increased average energy. The advantage of using the model is conserving the energy at the sleep procedure with the inactive nodes. If the fault occurs in the path then the alternative path with the next highest probability available is selected which will be delayed by the routing problem.

In cyber-physical system with energy efficiency and real-time for data transmission requires data aggregation tree construction as discussed in [15] and forms the required framework. The learning automata based degree-bounded bottle-neck data aggregation tree (DBBDAT), Reward Algorithm is used in developing the model. The learning process of DBBDAT construction and accuracy of different learning rates and thresholds, Time consumption, average solution time and average accuracy is enhanced and the nodes interacts 
with its predecessors and successors, that provides the optimal solution with the tradeoff between accuracy and overhead that can be controlled at the established framework as discussed in Buratti et. al., [16].

The network causes delay with an increase in the density of the vehicles on road resulting in route jam in the network and can be estimated using the collaborative learning automata as described in [17]. The learning Automata stationed at the nearest access points is accessible by the cluster that provides the system model with route selection and collaborative information sharing with density estimation. The rescue operations for dense urban regions using VSNs is effective and the effectiveness of the solution on the real test beds with changing environments.

Youcef et. al., [18] proposed a model checking an enhanced version of the carrier sense multiple access with collision avoidance protocol that models an enhanced variant of the IEEE 802.11. The CSMA/CA protocol with UPPAAL model checking tool provides the UML state machine of a wireless station and its back-off procedure. Thus, the safety properties are satisfied using this model while finding the quantitative annotations is quite difficult.

The communication process for the operation of a different protection scheme in a network requires a circuit breakers that combines the networks and transmission scheme. The Automatic translation of vendor-supplied specifications and simulations are of user interest where the protocols are used to monitor and send command to such devices as stated in [19].

Sudip et. al., [20] described the network with interlinked network objects and traditionally internetworked systems that are applicable for a heterogeneous network. The CPS networks equipped with the learning automata is used in the topology of the cyber-physical system. The comparison of DCF, VBA and LAVBA in terms of data loss, delay, delivery ratio and throughput is estimated. The model increases the efficiency of data transmission with sequence number individually. While these works look feasible with the different distributions of the $\mathrm{k}$ value that are currently operating in the network.

Networks supporting the environmental and underwater equipment monitoring can be made adaptive to data broadcasting using the MAC and network layer protocols with adaptive push systems and Broadcasting algorithm as proposed in [21]. The Convergence of the automation of the task request probabilities, mean response drawn with data access provides the adaptive push system with acoustic information and dissemination of data. The high latency of the underwater acoustics is checked normally using these algorithms proposed in Carolina et. al., [22].

Cross-layer design and adaptive rate control for the downlink wireless transmission a downlink scheduling and rate selection is determined as described in [23]. This uses the adaptive downlink scheduling with the rate selection that enhances the probability of crossdetection with the best rate, SINR, time evolution and scheduling probabilities. Thus, adaptivity computes the best transmission rate and is suitable for pedestrian and low mobility applications in [24] that uses time sharing and ARPANET process to TELNET protocol which is the mother of all protocols.

Al Agha et. al., [25] proposes a Self-manageable and adaptive industrial cyber-physical systems that uses service oriented architecture with the help of autonomic service management. The proposed algorithm utilizes the Service-Oriented Architecture which is described in Autonomic Service Manager Design for IEC61499. The advantage of using SQWRL with query engine enables the intelligent self-management properties with selfoptimization and resource utilization as their performance analysis. The proposed algorithm fails with the cognitive learning of the node behavior. 
Da et. al., [26] address the Network Autonomic Management that are conceptual, functional with related practical issues involved in operation, administration and management of the autonomic computing environment. The Autonomicity, Adaptability Degree, Intelligence degree, Awareness Degree can be strengthened using Autonomicity while the Memory Strength, Heterogeneous Functionality Management and Administrator permissions would be difficult while configuring the network.

The flexible two-level communication layer and three complimentary nodes can be used in Simple Object Access Protocol as discussed in [27]. Robert et. al., [30] discussed the selfoptimizing architecture that balances the goals of maximizing the process services requests in a service oriented environments. The network is configured with a content based routing involving network economics, congestion pricing, optimal routing and flow control. The proposed method does not estimate the derivatives of the congestion prices in an environment.

IEEE 802.15.4 wireless standard is used to implement the low cost, low-power wireless sensor platform implemented and can be utilized to design the compact wearable sensors for long-term measurement of electro-thermal activity, temperature, motor activity and photoplethysmography using i-Calm proposed by Fletcher et. al., [31]. This supports in gathering data in naturalistic settings with effective computing and health monitoring. The autonomic and affective disturbances for the growing number of nodes is widely affected in the larger network.

\section{BACKGROUND WORK}

Misra et. al., [14] focuses on the classification of the MAC protocols according to the conventional MAC techniques. But the classification does not find the MAC protocol which are suitable for the given set of conditions. The right MAC layer can be chosen by classifying congestion control using automaticity. The dynamic environment contains, a) limited use of sensing range b) unknown target distribution and c) the observation of large area. Because of all these conditions sensors are unable to cover the entire range of interest. Therefore, fixed sensor locations are not sufficient. It is preferred to be sensors that has to move dynamically with respect to the movement and distribution of targets and other sensor to maximize coverage.

In recent research, sensor network mobility discloses that mobile sensor can self-organize to provide good coverage than static sensors. The existing applications use only uninform mobility [16].

Thus, this problem may be considered as that of learning automata to co-ordinate the sensor target motion in continuous workspace and also a high level task allocation problem by dividing the workspace into discrete regions such that each region is allocated a group or coalition of sensor to track the target with,[22] [23].

\section{PROBLEM DEFINITION}

\section{Problem Statement}

In a dynamic environment the sensor nodes are deployed randomly while in static environment the sensor nodes are fixed in a particular pattern. While connecting ' $n$ ' number of dynamic nodes in a Wireless Sensor Network using ultra-wide band causes more congestion and reduces the packet delivery ratio. This occurs due to actuation of necessary information that are communicated through the various wireless sensor nodes. 


\section{Objectives}

Collision is one of the common parameter in addressing the issue in Wireless Sensor Networks. The collision is due to the congestion between the large numbers of nodes in a dynamically created environment. Thus, LA based approach is used to adaptively make the data packet arrival rate and service rate to avoid the congestion between the nodes.

The objectives of the work are to:

- To increase the packet delivery ratio with reducing energy consumption and rate of collision.

- Increase the throughput and the fault tolerance level at the Unicast and Multicast dynamically created environment.

\section{Assumptions}

- Autonomic Network Architecture is considered at the MAC Layer of the WSN for a dynamic environment with 500-1000 sensor nodes.

- The sensor nodes can either behave as a Master/Slave in a pre-defined dynamic environment.

\section{SYSTEM AND MATHEMATICAL MODEL}

Collision is one of the common parameter in addressing the issue in Wireless Sensor Networks. The collision is due to the congestion between the large numbers of nodes in a dynamically created environment. Thus, LA based approach is used to adaptively make the data packet arrival rate and service rate to avoid the congestion between the nodes.

\section{Local Automate Utility Management}

In our work the Local Automate (LA) based approach is considered for congestion control in WSN that improves the packet delivery ratio and the fault tolerance levels. The congestionavoidance using ANA architecture is proposed using the preliminaries of the LA concepts [26].

The autonomic architecture comprises of Medium Access Control Layer designed with Learning Automata Utility Management (LAUM). The architecture is designed based on the stochastic model and process reduces the overall design complexity with hassle-free deployment of the network involved with large number of sensor nodes. In this paper, the adaptive automata frequently uses the term "learning" that relates to the act of understanding based on the experience acquired and altering behavior of the network. Thus, in the adaptive automata adapts through a series of interactions within them to the dynamic environmental responses [27].

In optimizing the problems using the LA a best set has to be considered wherein, learning with high level of uncertainty with automaton will be a suitable choice in WSNs [31].

The source control mechanism is to transmit the data to the sink node or destination node. If congestion is detected, then the sink node has to inform the source to control its rate of transmission of packets [23]. For example, in hop-by-hop back pressure mechanism, the intermediate nodes inform the source to adjust the rate of flow of data dependency on the congestion.

In the proposed method, ANA controls the rate of flow of data easily including intermediate node and sink node. In this case, intermediate node does not provide any feedback to source node. On the other hand, intermediate node adjusts themselves depending on the output of automaton and continuously interacts with the environment [26]. This makes the nodes to select the rate of transmission of packets. By comparing with hop-by-hop back pressure mechanism and existing source control mechanism, the proposed method increases 
the efficiency of the network by optimizing the unwanted consumption of energy at the nodes and the link capabilities.

\section{Mathematical Model}

The probability of the sensor appearing for a short time is defined as

$P(1: \delta t)=\beta(\delta t)$

where, $\beta$ is the constant that defines the priority level of the sensor node with MASTER/SLAVE (1/0)

The probability of ' $n$ ' tasks that occur in a path is provided with

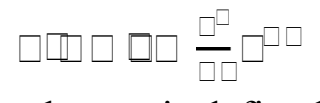

where, $r$ is defined by

The mean distance of the source and the destination node is calculated as

$<n>=r$

Let $\delta$ t be the small time interval in the total duration ' $t$ ' of the event that occurs. The probability of considering the learning based local automata would be $\beta(\delta \mathrm{t})$ and that of its absence is the $1-\beta(\delta t)$.

For the LACAS based ANA architecture let us estimate the probability with the condition $\beta(\delta \mathrm{t}) \rightarrow 1$.

The product of the two probability with its MASTER/SLAVE status is given by

$\mathrm{P}(0: \mathrm{t}+\delta \mathrm{t})=\mathrm{P}(0: \mathrm{t})(1-\lambda \delta \mathrm{t})$

Solving the said zeroth order differential equation reduces to

$\mathrm{P}(0: \mathrm{t})=\mathrm{ke}^{-\lambda \mathrm{t}}$

The constant ' $k$ ' in equation 6 would be assigned with the maximum limit of 1 and the exponential vary in the range of $0<\mathrm{e}^{-\lambda \mathrm{t}}<1$.

The probability in finding the neighborhood would be solving a second order differential equation computing for the present and future samples of the disturbed path.

$\mathrm{P}(\mathrm{n}: \mathrm{t}+\delta \mathrm{t})=\mathrm{P}(\mathrm{n}: \mathrm{t})(1-\lambda \delta \mathrm{t})+\mathrm{P}(\mathrm{n}-1: \mathrm{t}) \lambda \delta \mathrm{t}$

Equation 7 can be addressed as the first order differential equation as,

$d P(n: t)+\lambda P(n: t)=\lambda P(n-1: t)$

Solving the first order differential equation results in 
$\mu(t)=e^{\lambda t}$

and the probability is deduced as

$\mathrm{P}(1 ; \mathrm{t})=\lambda \mathrm{te}^{-\lambda \mathrm{t}}$

Therefore, for ' $n$ ' number of nodes present in the LACAS based ANA environment is $\mathrm{P}(\mathrm{n}+1: \mathrm{t})=(\lambda \mathrm{t})^{\mathrm{n}+1} /\left((\mathrm{n}+1) ! * \mathrm{e}^{\lambda t}\right)$

The design does not effects the mean of the distance and is given by

$<n>=r$

The variance determined in the said problem is unchanged and is given by $\sigma=r^{1 / 2}$

\section{Implementation}

The sensor node configuration and its implementation is created in an environment as shown in Figure 2(a) along with the parameters chosen as described in Table I. The network considered is as shown in Figure 2(a). The density of the sensor nodes are concentrated at the centroid and with lower densities as it moves out of the centroid. Figure 2(b) describes the part of the centroid of Figure 2(a). The large number of nodes varying from 50-100 sensor nodes form major cluster nodes as indicated with light colored hexagonal boxes. In our implementation, the light colored hexagonal boxes are considered as Master and remaining dark colored circles as slaves. While the darkened circular nodes are purposely made failure during execution so that packet loss has to occur. The created environment maps with the Table I specifications and the detailed process is explained in next section.

The process of involving the LA based ANA in the MAC layer of the UWD seems to be quite difficult in handling the large packets due to which the throughput, energy consumption and fault tolerance levels would be expected to be high. While the process of everyone MASTER/SLAVES would be one of the feasible solution in handling the said parameters and can convince the user requirements.

\section{LA based Model with ANA}

For the analysis of LA based ANA model we have considered each packet having a size of 2048 and 4048 for smaller and larger scales respectively. The estimation of packet delivery rate is considered in presence of atleast five Master nodes and all others acting as an intermediate nodes without change in source and sink nodes. While in Taleb et., al, [39] considered 1024 packet size with only one MASTER node.

In normal mode of propagation the packet size remain the same during the communication between the smaller till the larger number of nodes without any change in node density. With LACAS the congestion would be compromised once applicable to the normal mode of propagation but found unrealistic when applied with the larger density nodes at the considered smaller area. The LA-ANA is evaluated in the above said scenarios that is feasible in reducing the congestion [1][15][31].

Let us consider the large number of nodes as shown in Figure 2(a). In Figure 2(b) the boxes shows the MASTER, hexagonal boxes with the routing paths and nodes 6 and 33 are the accidental nodes or the breakdown nodes. The 8,50 and 41 are monitoring the status in 
propagating the data packets between the source and the destination indicated with larger rings. Few of the nodes that are not closer to the source and destination are also active that shows the neighborhood of the nodes and the key concept of LA based ANA architecture.

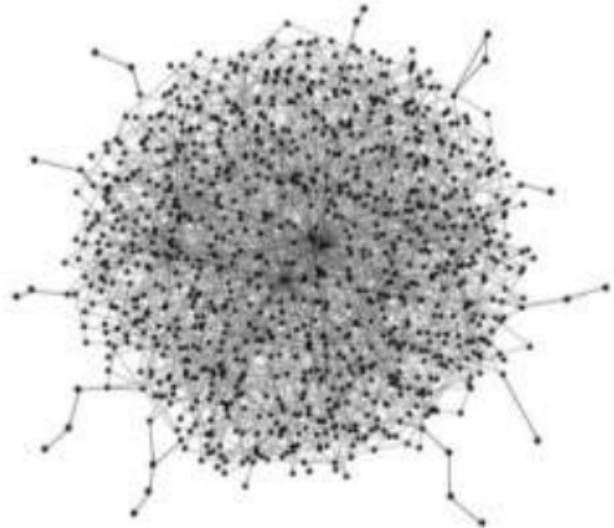

Figure 2(a). Sensor Nodes of 1000

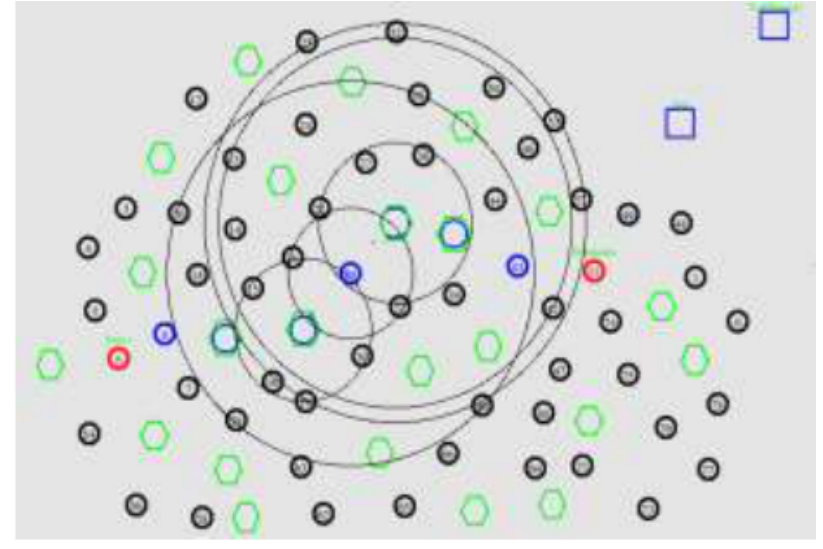

Figure 2(b). Large number of nodes varying from 50-100 sensor nodes

The actual process starts at this particular part with the large data sent in a dynamically large environment. The considered AODV is as simple and the intermediate nodes are considered throughout the environment. The probability of occurrence of the intermittent nodes along with the induced node is calculated using the equation (6). Furthermore, the optimal routing along with the selection of the randomly distributed nodes are chosen that finds the complete environmental response of failure nodes at that junction.

\section{Algorithm 1: LA-ANA in UWB}

Step 1: Consider the AODV environment with large number of nodes in an dynamic WSN environment.

Step 2: Add the intermediate nodes between the source and destination

Step 3: Determine the probability of occurrence P [ ] of each node induced.

Step 4: Choose the selected action for the tasks assigned with determining the probability of selecting the randomly distributed nodes.

Step 5: The optimal task is chosen in routing between intermittent and updates the selected route.

Step 6: The environment response is updated along with the task chosen using the Local Automate; LACAS( ).

Step 7: If the condition fails in updating to the newer value the previous states probabilities are recalled.

Step 8: Now transmit the packet only till the MAC layer of the network

Step 9: If error found with the occurrence then the process is repeated from step 5

Step 10: $\quad$ Now the process happens based on the LA-ANA architecture.

The LA-ANA uses the MASTER/SLAVE concept wherein the node before entering to the LACAS would be at the SLAVE. Once the same node attains the probability of turning $\beta(\delta t)$ $\rightarrow 1$ then at the outset it is turned to MASTER. Thus, turning the node itself into SLAVE at the initial phase and resulting to MASTER after the interference of LA-ANA at the second phase. The said operation may be vice-versa based on the neighboring nodes. 


\section{Algorithm 2: LACAS in UWB environment}

Step 1: The defined environment along with the generic nodes are considered.

Step 2: The probabilities of the primary routed nodes along with the neighboring nodes are tabulated

Step 3: The estimation of the cost at the respective nodes wrt the task assigned are calculated based on the probabilities avail from Step 2.

Step 4: The local automata is now complete for the selected network

The local automate provides a complete network that is feasible for propagation with more packet delivery rate. If in case the LACAS selected path fails the complete network is not disturbed and the usage of the neighboring nodes exists. The process of estimating is followed in equation (11) wherein involving the $(n+1)$ th samples at this moment of time. That is, ' $n$ ' number of MASTERS/SLAVES. The process continues until the occurrence is fulfilled. If in any case, any other intermediate error would occur in the process the same $(n+1)$ th samples are recalculated at that instant of time.

The LA-based model with ANA in MAC Layer is used in our work for congestion control in WSNs is described in Algorithm 1. Algorithm 2 depicts the availability of LACAS that has been considered while implementing LA based ANA for a WSNs environment at the MAC layer.

The algorithm referred predicts the probability of the each and every node present in the route and considers the best node. The probability of the selection of the nodes are determined by the first and second order differential equations that are deduced in the Mathematical model section. The task assigned and calculated at the LA-ANA and LACAS are depicted in sequential flow chart shown in Figure 2(c).

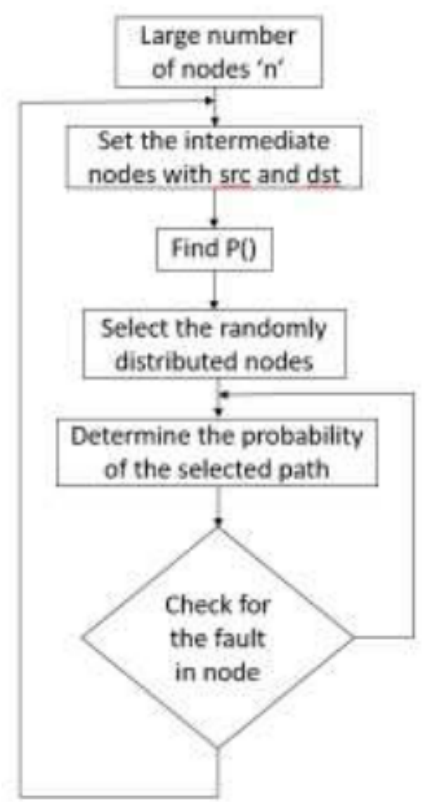

Figure 2(c). Sequential Flowchart for LA-ANA and LACAS

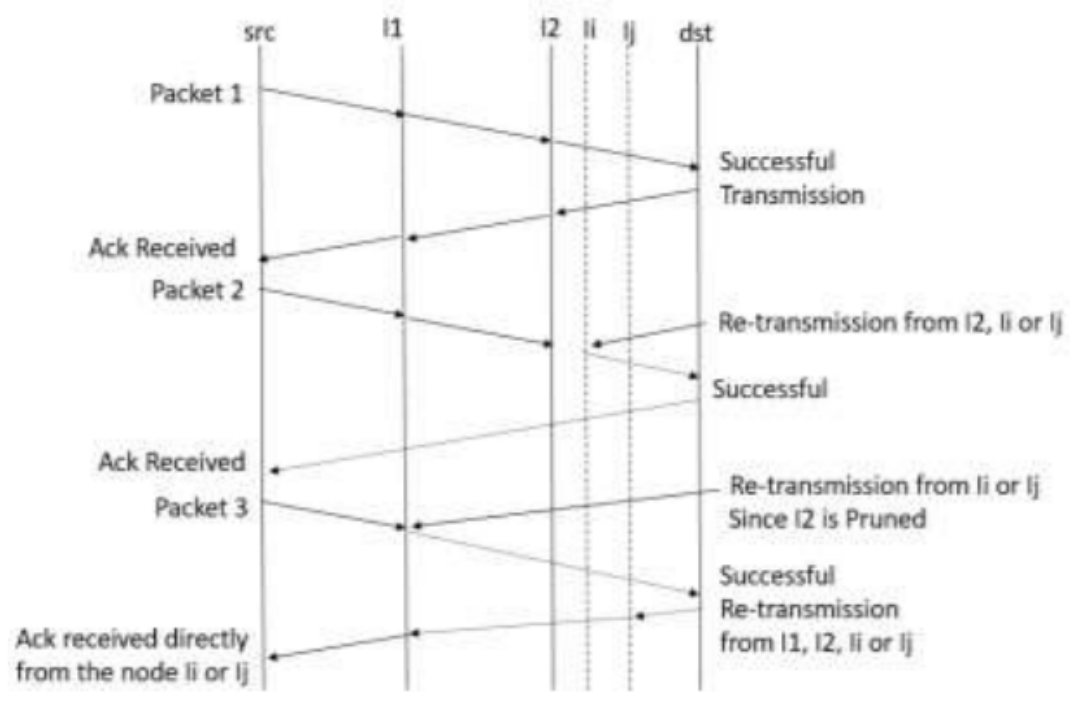

Figure 2(d). Timing diagram for transmission of packets

\section{Timing Diagram}

The transmission of packets in the LA-ANA based architecture is as shown in Figure 2(d) with and without loss of packets. Transmission of only three packets are considered for the representation of the timing diagram. The I1 and I 2 are the intermediate nodes wherein the 
successful re-transmission occurs even if the packet loss occurs at the Intermediate node I2. During the acknowledgement process if the node $\mathrm{I} 2$ fails the data is retransmitted from the I1 that proves the successful implementation of the LA based ANA architecture in an UWB environment. If in case both the intermediate nodes fails then there exist the rerouting path Ii or Ij with their neighborhood that successfully transmits the data packets and is shown with the dotted lines at the two levels.

\section{SIMULATION AND PERFORMANCE EVALUATION}

\section{Simulation Environment}

The environment is set-up in an UWB range operating at $10.6 \mathrm{GHz}$ with the double the power density propagation limit. The cause in increasing the power density is to make $\mathrm{Tx} / \mathrm{Rx}$ more reliable for the packets transmitted that increases the reception level. The data transmitted in the range are less susceptible to the noise levels due to the smaller space and the chances of having interference would be high due to the larger node density. The probability of assigning the random number for the complete set of nodes that are chosen with a lesser value and is around 5. The path loss is considered for a level of 2 to distinguish our proposed algorithm with the available LACAS. The simulation is performed in NS2.31 simulator. The remaining simulation parameters that are considered in simulating the LA-ANA based approach in an UWB environment is given in Table I.

Table 1: Simulation Parameters

Simulation Time: 50 secs. Temperature: 300.0 Kelvin Dimension: $(1 \mathrm{~km}, 800 \mathrm{~m})$ Number of Nodes: 100-1000Node Placement : Random Propagation limit: $-111.0 \mathrm{dBm}$ Propagation path loss: Two ray

\author{
Noise Figure: 10.0 \\ Seed for generation of random numbers: 5 Terrain \\ Radio Type : Radio-Accnoise (standard radio model) \\ Radio Rx type: SNR bound \\ Radio Tx power: $20 \mathrm{dBm}$ \\ MAC protocol: 802.11 \\ Architecture: Learning Automate based Autonomic \\ Network Architecture
}

The simulation time chosen is for $50 \mathrm{sec}$ with the seed for generating the number is limited to 5 terrain. The terrain provides the information of 5 levels of difficulties in reaching the randomly distributed nodes that are set with the priority wherein 5 being the least. The number of nodes are varied between 10-1000 nodes creating a larger network with limiting to $1 \mathrm{Km}$ and $800 \mathrm{~m}$ range. The sender nodes that are in dynamic environment are immobile during for a particular dynamic pattern. The propagation limit is set to $-111.0 \mathrm{dbm}$ that proves greater the same would be possible for our proposed algorithm. The noise figure is chosen bit higher than the LACAS and is about 10.0 in our proposed work. The standard operating temperature of 300.0 Kelvin is considered. The standard radio model is preferred in an UWB with SNR bound type of receiver. The transmission power is increased to $20 \mathrm{dBm}$ due to the large number of nodes present in a lesser dimension.

\section{Performance Metrices}

The variation in the packet delivery in considered and evaluated for the throughput, energy consumption, collisions, packet delivery ratio, unicast and multicast delivery along with the fault tolerance. 


\section{Performance Analysis}

The levels of collision are bit far due to the switching of MASTER/SLAVE concept helping the source and sink nodes that propagates at the bit-faster rate making the LACAS-UWB to have less collisions as shown in Figure 3. Thus, the number of collisions per unit area is more effective with a reduction of $30-40 \%$ as the number of nodes increases per unit area.

The transmission of large data at the faster rate would lead to more collision and may lead to propagation delay. In Figure 4 the delay (ms) is reduced by an optimal ratio of $15 \%$ compared with LACAS. It is due to the reduced number of collisions with increased number of nodes. The delivery rate is also increased due to the lesser collisions and neighborhood that made the transmitter and receiver to choose separately. The ratio was increased by an amount of $20 \%$ as shown in Figure 5 with reduced fluctuations as seen in LACAS.

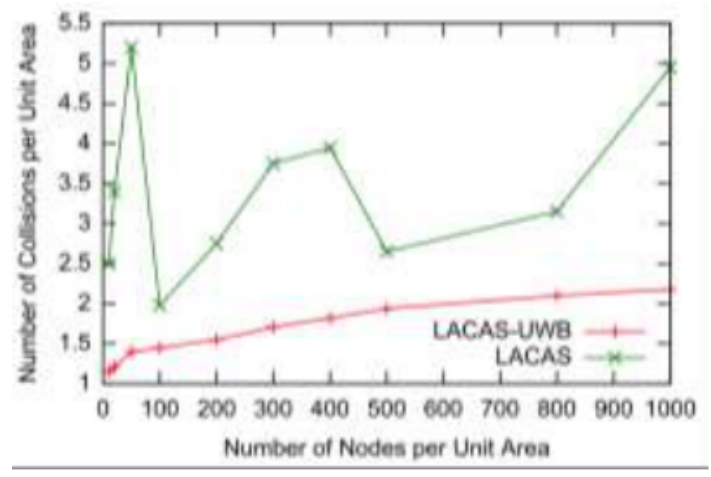

Figure 3. Number of collisions

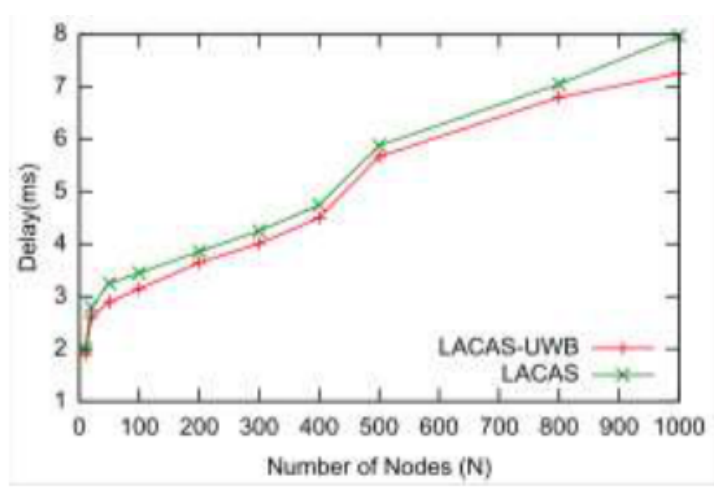

Figure 4. Congested mode with LACAS and LACAS-UWB

The unicast and multicast with LACAS-UWB provides optimal time required in delivering the packets transmitting from the source to the destination nodes due to fluctuation in the collisions in LACAS that lead to the decreased packet received rate at the LACAS. Thus, reducing the queuing of packets in the LACAS-UWB as shown in Figure 6 and Figure 7 for Unicast and Multicast delivery, respectively.

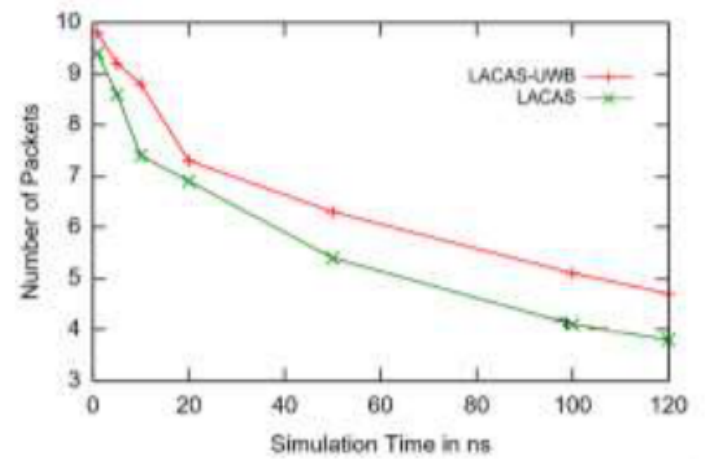

Figure 5. Packet delivery ratios with the Transmitter and Receiver chosen separately

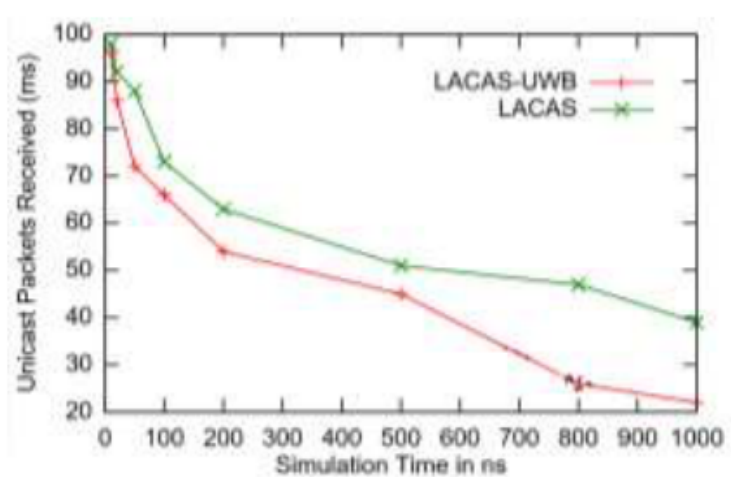

Figure 6. Unicast delivery 


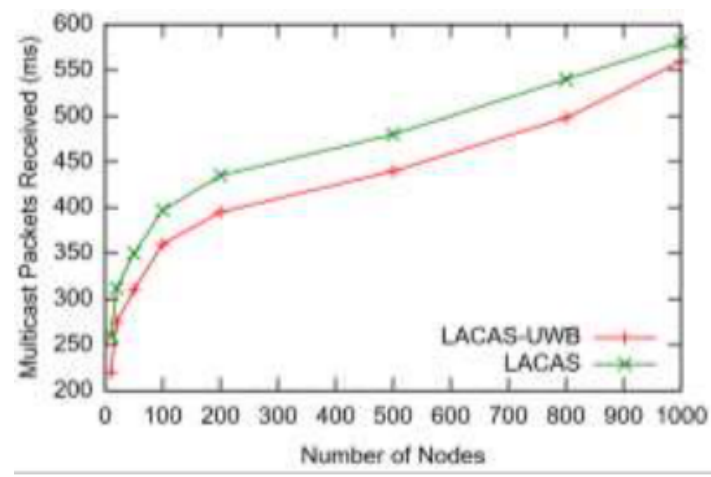

Figure 7. Multicast delivery

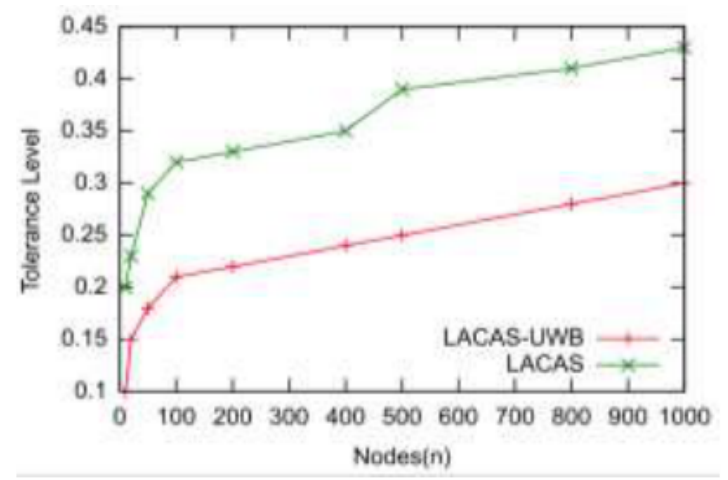

Figure 8. Fault Tolerance

The fault tolerance level at the MAC level is measured as the number of packets that transverse through a path from the various layers of the protocol stack of the corresponding nodes. The LACAS would not significantly affect the functionalities of the MAC Layer corresponding to the flow of signals through it thus LACAS-UWB would be an optimal algorithm in increasing the fault tolerance level as shown in Figure 8.

\section{CONCLUSIONS}

In this paper, a LA-ANA using the cascode architecture is designed for UWB environment. The concept is mathematically analyzed and the performance results are compared through simulation. The proposed algorithm is capable of effectively avoiding the congestion. Simulation results shows that the packet delivery ratio is increased consuming less amount of energy that is available at the nodes and decreases the number of collisions at the intermediate nodes.

The benefit of our suggested algorithm using LA-ANA is that the local automata in the various sensor nodes are stationed and helps in communicating with locally optimal tasks at each moment. Thus, understanding of past congestion concentrations leads to global optimal solution to reduce the congestion in the future. The proposed algorithm would provide an effective 30-40\% improvement in handling the nodes at every levels of the congestion without disturbing the complete network layer of the WSN.

\section{REFERENCES}

[1] H. Grichi, O. Mosbahi, M. Khalgui and Z. Li, "RWiN: New Methodology for the Development of Reconfigurable WSN", IEEE Transactions on Automation Science and Engineering, 14(1): 109-125, 2017.

[2] F. Verbeek, P. Yaghini, A. Eghbal and N. Bagherzadeh, "Deadlock Verification of Cache Coherence Protocols and Communication Fabrics", IEEE Transactions on Computers, 66(2): 274-284, 2016.

[3] T. Vollmer, M. Manic and O. Linda, "Autonomic Intelligent Cyber-Sensor to Support Industrial Control Network Awareness", IEEE Transactions on Industrial Informatics, 10(2): 1647-1658, 2014.

[4] H. Wang, N. Agoulmine, M. Ma and Y. Jin, "Network Lifetime Optimization in Wireless Ssensor Networks", IEEE Journal on Selected Areas in Communications, 28(7): 11271137, 2010. 
[5] K. Cohen and A. Leshem, "A Time-Varying Opportunistic Approach to Lifetime Maximization of Wireless Sensor Networks", IEEE Transactions on Signal Processing, 58(10): 5307-5319, 2010.

[6] P. Bohm, "Incremental and Verified Modeling of the PCI Express Protocol", IEEE Transactions on Computer-Aided Design of Integrated Circuits and Systems, 29(10): 1495-1508, 2010.

[7] J. Kay and J. Frolik, "An Expedient Wireless Sensor Automaton With System Scalability and Efficiency Benefits", IEEE Transactions on Systems, Man, and Cybernetics - Part A: Systems and Humans, 38(6): 1198-1209, 2008.

[8] S. Koestner, D. Breton, D. Charlet, F. Fontanelli, M. Frank, C. Gaspar, G. Haefeli, R. Jacobsson, B. Jost, G. Mini, N. Neufeld, R. Nogueira, C. Potterat, P. Robbe, M. Sannino and I. Videau, "Generic and Layered Framework Components for the Control of a Large Scale Data Acquisition System", IEEE Transactions on Nuclear Science, 55(1): 362-369, 2008.

[9] S. Drago, F. Sebastiano, L. Breems, D. Leenaerts, K. Makinwa and B. Nauta, "ImpulseBased Scheme for Crystal-Less ULP Radios", IEEE Transactions on Circuits and Systems I: Regular Papers, 56(5): 1041-1052, 2009.

[10] J. Misic, S. Shafi and V. Misic, "Maintaining Reliability Through Activity Management in an 802.15.4 Sensor Cluster", IEEE Transactions on Vehicular Technology, 55(3): 779$788,2006$.

[11] S. Misra, V. Tiwari and M. Obaidat, "Lacas: Learning Automata-based Congestion Avoidance Scheme for Healthcare Wireless Sensor Networks", IEEE Journal on Selected Areas in Communications, 27(4): 466-479, 2009.

[12] M. Thathachar and V. Phansalkar, "Convergence of Teams and Hierarchies of Learning Automata in Connectionist Systems", IEEE Transactions on Systems, Man and Cybernetics, 25(11): 1459-1469, 1995.

[13] Q. Zhao, L. Shen and C. Ding, "Stochastic MAC-layer Interference Model for Opportunistic Spectrum Access: A Weighted Graphical Game Approach", IEEE Journal of Communications and Networks, 18(3): 1229-2370, 2018.

[14] S. Misra, P. Krishna, V. Saritha, H. Agarwal, A. Vasilakos and M. Obaidat, "Learning Automata-Based Fault-Tolerant System for Dynamic Autonomous Unmanned Vehicular Networks", IEEE Systems Journal, 11(4): 2929-2938, 2017.

[15] A. Koubaa, R. Severino, M. Alves and E. Tovar, "Improving Quality-of- Service in Wireless Sensor Networks by Mitigating "Hidden-Node Collisions", IEEE Transactions on Industrial Informatics, 5(3): 299-313, 2009.

[16] C. Buratti and R. Verdone, "Performance Analysis of IEEE 802.15.4 Non-Beacon Enabled Mode", IEEE Transactions on Vehicular Technology, 58(7): 3480-3493, 2009.

[17] N. Kumar, S. Misra and M. Obaidat, "Collaborative Learning Automata-Based Routing for Rescue Operations in Dense Urban Regions Using Vehicular Sensor Networks", IEEE Systems Journal, 9(3): 1081-1090, 2015.

[18] Y. Hammal, J. Ben-Othman, L. Mokdad and A. Abdelli, "Formal Modeling and Verification of an Enhanced Variant of the IEEE 802.11 CSMA/CA Protocol", Journal of Communications and Networks, 16(4): 385-396, 2014.

[19] C. McParland, S. Peisert and A. Scaglione, "Monitoring Security of Networked Control Systems: It's the Physics", IEEE Security \& Privacy, 12(6): 32-39, 2014.

[20] S. Misra, P. Krishna, V. Saritha, H. Agarwal, Lei Shu and M. Obaidat, "Efficient Medium Access Control for Cyber-Physical Systems With Heterogeneous Networks", IEEE Systems Journal, 9(1): 22-30, 2015. 
[21] P. Nicopolitidis, G. Papadimitriou and A. Pomportsis, "Adaptive Data Broadcasting in Underwater Wireless Networks", IEEE Journal of Oceanic Engineering, 35(3): 623-634, 2010.

[22] C. Pinart, "A Multilayer Fault Localization Framework for IP Over All-Optical Multilayer Networks", IEEE Network, 23(3): 4-9, 2009.

[23] M. Haleem and R. Chandramouli, "Adaptive Downlink Scheduling and Rate Selection: A Cross-Layer Design", IEEE Journal on Selected Areas in Communications, 23(6): 12871297, 2005.

[24] W. Dai, V. Dubinin, J. Christensen, V. Vyatkin and X. Guan, "Toward Self-Manageable and Adaptive Industrial Cyber-Physical Systems With Knowledge-Driven Autonomic Service Management", IEEE Transactions on Industrial Informatics, 13(2): 725-736, 2017.

[25] K. Al Agha, M. Bertin, T. Dang, A. Guitton, P. Minet, T. Val and J. Viollet, "Which Wireless Technology for Industrial Wireless Sensor Networks? The Development of OCARI Technology", IEEE Transactions on Industrial Electronics, 56(10): 4266-4278, 2009.

[26] R. Bezerra and J. Martins, "Network Autonomic Management: A Tutorial with Conceptual, Functional and Practical Issues", IEEE Latin America Transactions, 12(2): 306-314, 2014.

[27] T. Vollmer, M. Manic and O. Linda, "Autonomic Intelligent Cyber-Sensor to Support Industrial Control Network Awareness", IEEE Transactions on Industrial Informatics, 10(2): 1647-1658, 2014.

[28] N. Van Wambeke, E. Exposito, C. Chassot and M. Diaz, "ATP: A Microprotocol Approach to Autonomic Communication", IEEE Transactions on Computers, 62(11): 2131-2140, 2013.

[29] R. Boutaba, J. Martin-Flatin, J. Hellerstein, R. Katz, G. Pavlou and C. Lea, "Recent Advances in Autonomic Communications [Guest Editorial]", IEEE Journal on Selected Areas in Communications, 28(1):1-3, 2010.

[30] R. Callaway, M. Devetsikiotis, Y. Viniotis and A. Rodriguez, "An Autonomic Service Delivery Platform for Service-Oriented Network Environments", IEEE Transactions on Services Computing, 3(2): 104-115, 2010.

[31] R. Fletcher, K. Dobson, M. Goodwin, H. Eydgahi, O. Wilder-Smith, D. Fernholz, Y. Kuboyama, E. Hedman, Ming-Zher Poh and R. Picard, "iCalm: Wearable Sensor and Network Architecture for Wirelessly Communicating and Logging Autonomic Activity", IEEE Transactions on Information Technology in Biomedicine, 14(2): 215-223, 2010. 